\title{
The role of lower genital tract microflora and antenatal maternal risk factors in preterm labour
}

\author{
R. J. Fernando', I. M. R. Goonewardene ${ }^{1}$, A. Nagahawatte ${ }^{2}$ and S. Fernando ${ }^{2}$ \\ The Ceylon Journal of Medical Science 2001; 44: 41-51
}

\section{Summary}

Objective: To determine the role of lower genital tract microflora and antenatal maternal risk factors in pre term labour (PTL).

Design: A prospective cohort study.

Setting: University obstetric unit and the Department of Microbiology, Faculty of Medicine, University of Ruhuna, Galle, Sri Lanka.

Subjects: Two hundred and one pregnant women with periods of gestation (POG) less than 25 weeks, presenting for antenatal care.

Method: Antenatal maternal risk factors for PTL were documented and swabs from posterior vaginal fornix and endocervical canal were obtained at the booking visit and at a POG of 32 weeks. The microbiological findings at booking and at a POG of 32 weeks, and the antenatal maternal risk factors in each subject were correlated with the occurrence of PTL.

Results and Conclusions: Colonisation of the lower genital tract with probable anaerobic gram negative bacilli at booking and possibly at a POG of 32 weeks was a high risk factor for the occurrence of PTL. Teenage and low socioeconomic status were also high risk factors associated with PTL. Current multiple pregnancy showed a possible association with the occurrence of PTL.

Key words: Preterm labour, risk factors, lower genital tract, microflora.

\section{Introduction}

Preterm labour (PTL) resulting in the delivery of premature babies is a major factor contributing to perinatal morbidity and mortality worldwide. In developed countries it has been found to occur in $7-10 \%$ of all pregnancies $(1,2,3)$ and be responsible for approximately $75 \%$ of all perinatal deaths (1). An incidence of about $10-15 \%$ has been found in India $(4,5)$.

As much as $85 \%$ of all neonatal deaths not associated with congenital abnormalities have been associated with PTL (6). Furthermore approximately $6-7 \%$ of survivors of preterm birth have been shown to have some form of major handicap and this incidence has remained unchanged during the past two dacades $(7,8)$.

Identifying the high risk group susceptible to develop PTL may help to prevent the high incidence of perinatal morbidity and mortality due to prematurity. However various scoring systems based on epidemiological and clinical risk factors for PTL have proved insensitive and non specific (9-12). The best indicator for the prediction of PTL is a previous history of PTL but this is applicable only in muligravidae. PTL often occurs in association with other complications of pregnancy such as antepartum haemorrhage, multiple pregnancy and severe pregnancy induced hypertension. Although in a significant proportion no identifiable cause can be found, ascending infection from the lower genital tract has been identified as probably an important cause for PTL (13 -17).

The specific objectives of this study were:

to identify the pattern of lower genital tract microflora in pregnant women presenting for antenatal care before the third trimester,

to assess any change in the above, later in pregnancy,

1. Departments of Obstertics and Gynaecology,

2. Microbiology, University of Ruhuna, Galle, Sril Lanka. 
to determine whether there is any relationship between the specific microorganism/s detected, antenatal maternal risk factors and subsequent occurrence of PTL.

\section{Subjects and Methods}

At the university antenatal clinic of the Teaching Hospital Galle, 206 consecutive pregnant women who had a period of gestation (POG) less than 25 weeks at booking and who had not been treated with any antibiotics during their current pregnancy, were recruited for the study.

Ethical clearence for this study was obtained from the Faculty of Medicine, University of Ruhuna.

Assuming the incidence of PTL to be a approximately $10 \%$ and to tolerate a maximum sampling error of $5 \%$ the minimum sample size was calculated to be 139 (18). A larger sample of 200 subjects was planned in order to obtain more reliable data.

The objectives, procedure and the advantages of the study were explained and written consent obtained in Sinhala. A pre tested structured interview was used to obtain relevant information and to document the presence or absence of known risk factor for PTL (9-12). With the aid of a Cusco's bivalve vaginal speculum swabs were obtained from the endocervical canal and the posterior vaginal fornix.

\section{Material and equipment}

Sterile Cusco's bivalve specula and sterile cotton wool swabs were used to obtain the specimens. Culture media used were:

1. Chocolate agar

2. Blood agar (Oxoid)

3. Gonococcal selective medium (Oxoid) with Vancomycin, Colistin Sulphate, Nystatin and Trimethoprim

4. MacConkey agar (Oxoid)

5. Listeria enrichment broth and Listeria selective medium (Oxoid)

\section{Procedures and protocols}

The subjects were examined by the first author. If there was a doubt about the period of gestation, transabdominal ultrasonography was performed. The dates were corrected if a difference of more than two standard deviations was found between the dates obtained by the scan and the dates obtained by menstrual data. Five subjects who resisted the insertion of the Cusco's bivalve speculum were excluded leaving a sample of 201 subjects for study. Five women with death in utero, two being due to severe pregnancy induced hypertension and three with no apparent cause were also excluded from analysis.

Six specimens were obtained using sterile Cusco's bivalve speculum:

Swab from the endocervical canal, swab from the posterior vaginal fornix, direct smears from each of these two sites, Gonococcal selective medium plated with the endocervical swab and swabs from the endocervical canal and posterior vaginal fornix placed in Listeria selective broth. In addition, using a wire loop, secretions were taken onto a fresh slide. A drop of freshly prepared $10 \%$ potassium hydroxide was added on to it and smelled for any fishy odour. The six specimens were processed in the Department of Microbiology at the Faculty of Medicine, Galle.

The direct smears were stained by Gram's method and the types of organisms and the number of pus cells were noted. The swabs from the endocervix and the posterior vaginal fornix were each plated on chocolate agar, blood agar and MacConkey agar. Chocolate agar and gonococcal selective medium plates were incubated at $37^{\circ} \mathrm{C}$ in a candle jar with 5-10\% carbon dioxide $\left(\mathrm{CO}_{2}\right)$. The blood agar and the MacConkey agar plates were incubated aerobically at $37^{\circ} \mathrm{C}$. Following overnight incubation, plates were examined for the presence of colonies.

Gardnerella species was identified by a positive Amine test. If colonies were present, the organisms were identified using the Gram stain and biochemical tests. Staphylococci species were identified by the coagulase test. 
Since facilities for anaerobic cultures were not available, the Gram negative bacilli detected in the gram stained smear were assumed to be 'probable anaerobic species' if they failed to grow aerobically and in $5-10 \% \mathrm{CO}_{2}$ on blood agar, MacConkey agar and chocolate agar. The swab containing Listeria enrichment broth was incubated at $37^{\circ} \mathrm{C}$ and sub cultured on first, second and seventh days on Listeria selective medium and blood agar, and examined for the presence of Listeria species.

Each subject had routine antenatal care. At a POG of 32 weeks the swabs were repeated. In addition the weight gain, urine for albumin, presenting part and engagement, amount of liquor and uterine irritability were noted. A digital vaginal examination was performed to assess the effacement and dilatation of cervix. Based on Creasy's risk score system (9), each patient was given a score at a POG of 32 weeks.

The first two authors were not informed about the microbiological findings. If pathogens such as gonococci were detected it was decided to treat such subjects appropriately and to exclude them from the study.

The subjects were followed up and advised admission to the ward immediately if labour pains occurred or if there was spontaneous rupture of membranes (SROM). The first author was immediately informed when the subjects were admitted in PTL or with pre-term pre-labour SROM. In each of these patients after the routine obstetric examination a sterile speculum examination was performed and if pre-labour SROM was detected the leaking amniotic fluid was obtained for direct smear and Gram stain and culture. If there was no SROM, a digital vaginal examination was performed to confirm labour and the POG was documented.

Labour was diagnosed in the presence of one or more regular painful uterine contractions per 10 minutes and a fully effaced cervix in a primipara or more than $3 \mathrm{~cm}$ dilatation of the cervix in a multipara. Subjects were considered to have PTL if they required treatment with tocolytics or delivered before the completion of a POG of 37 weeks. If PTL was diagnosed the patients were managed according to the PTL management protocol of the unit. If labour was suppressed with tocolytics, the POG at delivery was also documented.

\section{Data analysis}

Outcome of the pregnancy was classified as Term Labour (TL) or PTL. Patients who required treatment for PTL and subsequently delivered at term were classified with PTL group. Risk factors for PTL and the microbiological findings at booking and at a POG of 32 weeks were correlated with occurrence of PTL and TL.

The computer statistical software package Epi Info Version 6 was used. Differences in proportions of variables were assessed by the Chi Square Test and the Fishers Exact Test where appropriate. The relative risks (RR) for the occurence of PTL in the presence of various risk factors were calculated.

\section{Results}

PTL occurred in $14(7.0 \%)$ and 13 of them resulted in preterm delivery while one responded to treatment with salbutamol and bed rest and subsequently delivered at term. The mean POG at preterm delivery was 32.8 weeks. One patient who had preterm labour SROM at a POG of 32 weeks, delivered 18 hours later. Her liquor amnii was found to be sterile on Gram stain and aerobic culture.

There was a significantly high rate of PTL among teenagers and among women whose monthly income was less than Rs. $5000 /=(p<0.001)$. However there was no significant relationship between parity and the onset of labour. Although most Sri Lankan women do not smoke, their husbands may be smokers. There was no relationship between the husband's smoking habit and PTL. None of the risk factors known to be associated with PTL showed a significant positive relationship. A past history of more than two previous abortions (RR 2.6, 95\% CI. 0.6-10.1, $\mathrm{p}=0.2$ ) and twins in current pregnancy (RR $5.095 \% \mathrm{CI}$ 0.9-26.7, $\mathrm{p}=0.2$ ) showed a possibe relationship with PTL. (Table 2). 
Table 1

Basic characteristics of the subjects $(n=196)$

\begin{tabular}{|l|c|c|c|c|}
\hline \multirow{2}{*}{ Characteristic } & \multicolumn{2}{|c|}{ TL (n=182) } & \multicolumn{2}{c|}{ PTL (n=14) } \\
\cline { 2 - 5 } & $\mathrm{n}$ & $\%$ & $\mathrm{n}$ & $\%$ \\
\hline 1. Maternal age (Years & 17 & 9.3 & 3 & $21.4^{*}$ \\
(a) <20 & 105 & 57.7 & 8 & 57.1 \\
(b) 20-29 & 60 & 33.0 & 3 & 21.4 \\
(c) 30-39 & 83 & 45.6 & 7 & 50.0 \\
2. Primipara & 99 & 54.4 & 7 & 50.0 \\
3. Multipara & 120 & 65.9 & 11 & $78.5^{*}$ \\
4. Monthly family income \\
<Rs. 5000/=
\end{tabular}

POG $=$ Period of Gestation

$\mathrm{SD}=$ Standard Deviation

$\mathrm{TL}=$ Term Labour

$\mathrm{n}=$ number of subjects

PTL $=$ Pre term Labour

${ }^{*} \mathrm{p}<0.001$ 
Table 2

Relative risks of maternal historical and antenatal risk factors associated with PTL

\begin{tabular}{|l|c|c|c|c|}
\hline Risk factors & $\begin{array}{c}\text { TL } \\
(\mathbf{n}=\mathbf{1 8 2})\end{array}$ & $\begin{array}{c}\text { PTL } \\
(\mathbf{n = 1 4 )}\end{array}$ & $\mathbf{R R ~ ( 9 5 \% \text { CI } )}$ & $\begin{array}{c}\mathbf{p} \\
\text { value }\end{array}$ \\
\hline 1. Maternal medical problems & 36 & 3 & $1.1(0.3-3.8)$ & 1.0 \\
\hline $\begin{array}{l}\text { 2. } \$ 2 \text { Previous first } \\
\text { timester abortions }\end{array}$ & 10 & 2 & $2.6(0.6-10.1)$ & 0.2 \\
\hline 3. Creasy's score $>10$ & 21 & 2 & $1.2(0.3-4.8)$ & 0.7 \\
\hline 4. First trimester bleeding & 6 & 1 & $2.1(0.3-16.8)$ & 0.4 \\
\hline 5. Twins in current pregnancy & 2 & 1 & $6.5(0.6-67.4)$ & 0.2 \\
\hline 6. Previous treatment \\
$\quad$ for genital tract infection
\end{tabular}

$\mathrm{TL}=$ Term Labour $\quad \mathrm{RR}=$ Relative Risk $\quad$ PTL = Preterm Labour

$95 \% \mathrm{CI}=95 \%$ Confidence Interval $\quad \mathrm{n}=$ number of subjects

Table 3

Association between microorganisms detected at booking, and PTL

\begin{tabular}{|l|c|c|c|c|}
\hline Organism & TL (n=182) & PTL(n=14) & RR(95\% CI) & $\begin{array}{c}\text { p } \\
\text { value }\end{array}$ \\
\hline Diphtheroids & 67 & 05 & $1.0(0.5-2.0)$ & 0.84 \\
\hline Coagulase negative Staphylococci & 23 & 02 & $1.1(0.3-4.3)$ & 0.69 \\
\hline Lactobacilli & 175 & 12 & $0.8(0.7-1.1)$ & 0.12 \\
\hline $\begin{array}{l}\text { Probable anaerobic gram } \\
\text { negative bacilli }\end{array}$ & 14 & 04 & $3.7(1.4-9.8)$ & 0.03 \\
\hline Gardnerella species & 01 & 01 & $13.0(0.9-19.7)$ & 0.14 \\
\hline Candida species & 28 & 02 & $0.9(0.2-3.5)$ & 1.00 \\
\hline Micrococci species & 09 & 01 & $1.4(0.2-10.6)$ & 0.53 \\
\hline
\end{tabular}

See Table 2 for meaning of abbreviations. 
Table 4

Association between microorganisms detected at a POG of 32 weeks, and PTL

\begin{tabular}{|l|c|c|c|c|}
\hline Organism & TL (n=182) & PTL(n=10)* & RR(95\% CI) & p value \\
\hline Diphtheroids & 68 & 05 & $1.3(0.7-2.56)$ & 0.5 \\
\hline $\begin{array}{l}\text { Coagulase negative } \\
\text { Staphylococci }\end{array}$ & 53 & 01 & $0.3(0.05-2.2)$ & 0.29 \\
\hline Lactobacilli & 179 & 10 & $1.02(1-1.04)$ & 0.9 \\
\hline $\begin{array}{l}\text { Probable anaerobic } \\
\text { gram negative bacilli }\end{array}$ & 05 & 01 & $3.6(0.5-28.3)$ & 0.28 \\
\hline Candida species & 39 & 01 & $0.5(0.07-3.6)$ & 0.69 \\
\hline
\end{tabular}

* 4 patients delivered prior to a POG of 32 weeks.

See Table 2 for meaning of abbreviations.

Table 5

Microorganisms detected at booking and at a POG of 32 weeks

\begin{tabular}{|l|c|c|}
\hline Organism & $\begin{array}{l}\text { positive percentage } \\
\text { at booking }\end{array}$ & $\begin{array}{l}\text { positive percentage } \\
\text { at 32 weeks }\end{array}$ \\
\hline Diphtheroids & 35.8 & 38 \\
\hline $\begin{array}{l}\text { Coagulase negative } \\
\text { Staphylococci }\end{array}$ & 12.4 & 28.1 \\
\hline Lactobacilli & 93.0 & 98.4 \\
\hline $\begin{array}{l}\text { Probable anaerobic gram } \\
\text { negative bacilli }\end{array}$ & 8.9 & 3.1 \\
\hline Gardnerella species & 0.9 & 0.5 \\
\hline Candida species & 14.9 & 21.9 \\
\hline Micrococci species & 4.9 & 0.5 \\
\hline
\end{tabular}


Gonococci, Listeria and Streptococci species were not isolated in any of these samples. The presence of probable anaerobic gram negative bacilli in early pregnancy showed a significant positive relationship with PTL (RR 3.9, 95\% CI 1.4-11.2, $\mathrm{p}=0.03$ ).

Although they did not reach statistical significance the following trends were observed. Gardenerella in early pregnancy (RR $13.0,95 \% \mathrm{CI}$ 0.9-197, $p=0.14$ ) and probable anaerobic gram negative bacilli at a POG 32 weeks (RR $3.4,95 \% \mathrm{CI}$ 0.5-23.01, $\mathrm{p}=0.3$ ) showed a possible relationship with PTL.

Commonest microorganism isolated in the lower genital tract at booking and at POG of 32 weeks was Lactobacilli species ( $93 \%$ and $98.4 \%$ respectively). The second commonest was Diphtheroid species ( $35.8 \%$ and $38 \%$ respectively). Coagulase negative Staphylococci were isolated in $12.4 \%$ at booking and it had significantly increased to $28.1 \%(p<0.01)$ at a POG of 32 weeks. Candida species were present in about $15 \%$ and $22 \%$ respectively. All these patients were asymptomatic and were not treated.

Pus cells were seen in $85.5 \%$ of the smears taken at booking and $79.1 \%$ at a POG of 32 weeks. Majority of them had about 5-10 pus cells per high power field. However clue cells were not seen even in the smears which had a positive amine test.

\section{Discussion}

Prevention of Pre term Delivery (PTD) continues to be a major goal in present day obstetrics. Two broad types of preventive approaches have been undertaken to achieve this goal. First is a population based strategy that attempts to control the determinants of PTL at a community level. The second is strategies to identity the women who are likely to develop PTL (19). Despite these approaches and advances in obstetrics there has been no documented decrease in the rate of PTD in the last two dacades $(8,20)$. However there has been a substantial decrease in the neonatal mortality, mainly due to the improved survival of preterm infants following the advent of neonatal intensive care units $(21,22)$. This has been accompanied with a large increase in the cost of care for premature infants (23).

In a developing country such as Sri Lanka the prevention of PTD would be much more cost effective than spending a large amount of money on pre term neonatal care. If this goal is to be achieved the risk factors for PTD which are relevant to Sri Lankan pregnant woman should be indentified. In our study we have tried to indentify several known risk factors for PTL in relation to pregnant women in our region. The rate of PTL in this study $(7 \%)$ is similar to the incidence reported in developed countries $(1,2,3)$, but relatively less compared to India $(4,5)$. Considering the known risk factors for PTL, poor socio-economic status, teenage pregnancy, history of more than two previous abortions and twins in the current pregnancy were significantly associated with PTL.

In a study of 17,000 pregnant women it was found that the rate of spontaneous PTL in social classes IV and V were about $50 \%$ greater than in social class I and II and was much higher among unmarried mothers (24). In our study too there was a significantly high rate of PTL among women who had a low family income. A similar association has been found with teenage pregnancies but not with parity (23). Our study too showed the same. The exact causes for the increased incidence of PTL in the lower socioeconomic classes and among the teenagers are sitill to be found. Stress has recently been postulated as a cause of PTL $(25,26)$.

Multiple pregnancy is well known to be associated with PTL (26). Although maternal smoking is known to be associated with a high incidence of PTL none of the subjects in this study smoked. Almost $60 \%$ of their husbands were smokers but we could not find any relationship between passive smoking and PTL.

The Risk Scoring Systems in predicting labour which had been popular earlier have been found to be of limited value (9-12). In our study too we observed the same (Table 2). 
Ascending infection from the lower genital tract is known to be associated with PTL and the mechanisms by which the microorganisms cause PTL have been postulated (28). The organisms found to be associated wiht the occurrence of PTL are Group B Streptococci (29), Neisseria gonorrhoeae (30), Bacteriods species $(31,32)$, Gardnerella species (11), Mycoplasma and Chlamydiae (34), anaerobes in general (35), Listeria (36) and Ureaplasma (37). However, two Cochrane systematic reviews on the role of antibiotics for preterm labour with intact membranes and absent membranes $(38,39)$ did not find any significant reduction of PTL rates or benefits to mother or neonate in the use of routine antibiotics in the absence of clinical infection. The recently published findings of the worldwide multi centre randomised control trial (ORACLE) also support this view (40).

In our study, the isolation of probable anaerobic gram negative bacilli species in early pregnancy showed a significant positive relationship with PTL, with a possible relationship if it was isolated at a POG of 32 weeks. Gardnerella species detected in early pregnancy also showed a possible association with PTL.

The sample size was calculated assuming the prevalence of PTL to be approximately $10 \%$. However the actual proportion of PTL in this study was only $7 \%$. Therefore the possible association of risk and protection observed in this study may have reached statistical significance with a larger sample.

Unfortunately the facilities for anaerobic culture and isolation of genital Mycoplasms and viruses were not available at the time of this study. Hence we are unable comment on their relationship to P.TL.

An interesting feature noticed in our study was the absence of Neisseria gonorrhoeae, Listeria species and Group B Streptococci in any of the subjects. This is significant and could have contributed to the relatively low rate of PTL in this study. However isolation of group B Streptococci require the use of selective media for optimum isolation and such media were not available for the study. In Sri Lanka relatively high isolation rates have been reported earlier (41).

Considering the general pattern of microorganisms in this study, Lactobacilli predominated throughout pregnancy. Excepting in the case of coagulase negative Staphylococci there was no significant change in the pattern from mid pregnancy to late pregnancy.

The occurrence of PTL is probably due to multiple factors. The exact causes and the mechanism of PTL is not clearly understood yet. Eastman's statement over four decades ago that "only when the factors causing prematurity are clearly understood can any intelligent attempt at prevention be made" (42) is still true today and is a challenge to the obstetrician at present.

\section{Conclusions}

The rate of PTL in our study was similar to that of developed countries but less than that of India. The absence of any evidence of Gonococci, Listeria species and Group B Streptococcal infection in our subjects may have contributed to this relatively low rate of $7 \%$.

Teenage and low socio economic status carry a significanty high risk of PTL while a current twin pregnancy could also be possible risk factors.

Colonisation of the lower genital tract with probable anaerobic gram negative bacilli in a early pregnancy led to a four fold increase in the risk of PTL. Gardnerella infection may also be a risk factor. A risk score system did not prove to be of value in identifying subjects at risk of having PTL.

\section{Acknowledgement}

Mrs. Mallika Peellawattage, Mrs. Anoma de Silva and Mrs. B. Keembiyahetty assisted with microbiological analysis. 


\section{References}

1. Fuchs F. Prevention of prematurity. American Journal of Obstetrics \& Gynaecology 1976; 126: 809-817.

2. Lockwood C.J. Recent advances in elucidating the pathogenesis of preterm delivery, the detection of patients at risk, and preventive therapies. Current Opinion in Obstetrics \& Gynaecology 1994; 6: 7-18.

3. McDonald H.M., O'Loughlin J.A., Jolley P., Vigneswaran R., McDonald P.J. Vaginal infection and Preterm labour. British Journal of Obstetrics \& Gynaecology 1991; 98: 427 435.

4. Bhargawa S.K., Singh K.K., Saxena B.N. Eds. A national collaborative study of identification of high risk families, mothers and outcome of their off springs with particular reference to the problem of maternal nutrition, low birth weight, perinatal and infant morbidity and mortality in urban and rural slum communities: an ICMR Task Force Study. New Delhi: Indian Council of Medical Research 1990; p50-55.

5. Das B., Gogoi M.D. A clinical study of preterm labour. Journal of Obstetrics \& Gynaecology of India 1986; 34: 300-306.

6. Rush R.W., Keirse M.J.N.C., Howat P. et al. Contribution of preterm delivery to perinatal mortality. British Medical Journal 1976; 2: 965968.

7. Jones R.A.K., Cummins M, Davies P.A. Infants of very low birth weight. A 15 year analysis. Lancet 1979; i: 1332-1335.

8. Moutiquin J., Valerie M., Oliver I. Preterm birth prevention: Effectiveness of current strategies. Journal Summaries of Obstetrics \& Gynaecological Communications. 1996; 15: 835-851.

9. Creasy R.K., Gummer B.A., Liggins G.C. System for predicting spontaneous preterm birth. Obstetrics \& Gynaecology 1980;55: 692695.

10. Iams J.D., Jhonson F.F., Creasy R.K.
Prevention of preterm birth. Clinics in Obstetrics \& Gynaecology 1988; 31: 566-615.

11. McGregor J.A., French J.I., Richter R. et.al. Antenatal microbiologic and maternal risk factors associated with prematurity. American Journal of Obstetrics \& Gynaecology 1990; 163: 1465-1473.

12. Main D.M., Richardson D., Gabbe S.G., Strong S, Weller S.C. Prospective evaluation of a risk scoring system for predicting preterm delivery in black inner city women. Obstetrics \& Gynaecology 1987; 69: 61-66.

13. Gravett M.G., Hummel D., Eschenbach D.A., Holmes K.K. Preterm labour associated with subclinical amniotic fluid infection \& bacterial vaginosis. Obstetrics \& Gynaecology 1986; 67: 229-237.

14. Hillier S.L., Martius J., Krohn M. et al. A case control study of choioamniotic infection and histologic chrioamniotis in prematurity. New England Journal of Medicine 1988; 319: 972-978.

15. McGregor J.A. Prevention of preterm birth. New initiatives based on microbial-host interaction. Obstetrics \& Gynaecology Survey 1988; 43: 1-14.

16. Minkoff H., Grunebaum A.N., Schwarz R.H. et al. Risk factors for prematurity and premature rupture of membranes: A prospective study of vaginal flora in pregnancy. American Journal of Obstetrics \& Gynaecology 1987; 136: 824-833.

17. Sweet R.L., Landers D.V., Walker C., Schachter J. Chlamydia trachomatis infection and pregnancy outcome. American Journal of Obstetrics \& Gynaecology 1987; 136: 824833.

18. Vaughan J.P, Morrow R.H. Manual of epidemiology for district health management. WHOPublication, Geneva: 1989. p175178.

19. Lumley J. The prevention of preterm birth: unresolved problems and work in progress. Australian Paediatric Journal 1988; 24: 101111. 
20. Iams J.D. Current status of prematurity prevention. Journal of American Medical Assocication 1989; 262: 265-266.

21. Lee K.S., Paneth N., Gartner L.M. et al. Neonatal Mortality: An analysis of the recent improvements in the United States. American Journal of Medicine 1980; 70: 15-21.

22. Williams R.L., Chen P.M. Identifying the sources of the recent decline in perinatal mortality rates in California. New England Journal of Medicine 1982; 306; 207-214.

23. Doyle L.W., Murton L.J., Kitchen W.H. Increasing the survival of extremely immature infants - at what cost? Medical Journal of Australia 1989; 150: 558-563.

24. Fredrick J., Anderson A.B.M. Factors associated with spontaneous preterm birth. British Journal of Obstetrics \& Gynaecology 1976; 83: 342-350.

25. Jones S.A., Challis J.R.G. Steroides, Corticotropin-Releasing Hormone, ACTH \& Prostaglandin interactions in the amnion and placenta of early pregnancy in man. Journal of Endocrinology 1990;125: 153-159.

26. Petraglia F., Coukos G., Volpe A., Gennzzani $R$., Vale $W$. Involvement of placental neurohormones in human parturition. Annals of New York Academy of Science 1991; 622: 331-340.

27. Whitfield C.R. Multiple pregnany. In Whitfield C.R ed. Dewhurst's text book of Obstetrics \& Gynaecology for postgraduates. Oxford, London. Blackwell Scientific Publications. 1985; p482-495.

28. Bejar R., Curbelo V., Davi S.C., Gluck L. Premature labour: bacterial source of phospholipase. Obstetrics \& Gynaecology 1981; 57: 479-482.

29. Lamont R.F., Fisk N. The role of infection in the pathogenesis of preterm labour. Progress in Obstetrics \& Gynaecology. 1993; 10: 135-158.
30. Regan J.A., Chao S., James L.S. Premature rupture of membranes, preterm delivery and Group B Streptococci colonisation of mothers. American Journal of Obstetrics \& Gynaecology 1981; 141: 184-186.

31. Handsfield H.H., Godson W.A., Holmes K.K. Neonatal gonococcal infection IOrgastric contamination with Neisseria gonorrhoeae. Journal of American Medical Association 1973; 225: 697-701.

32. Bobitt J.R., Hayslip C.C., Damato J.D. Amniotic fluid infection as determined by transabdominal amniocentesis with intact membranes in premature labour. American Journal of Obstetrics \& Gynaecology 1981; $140 ; 947-952$.

33. Miller J.M., Hill G.B., Welt S.I. et al. Bacterial colonisation of amniotic fluid in the presence of ruptured membranes. American Journal of Obstetrics \& Gynaecology 1980; 137: 451458.

34. Lamont R.F., Taylor-Robinson D., Wigglesworth J.S., Furr P.M., Evens R.T., Elder M.G. The role of Mycoplasmas, Ureaplasmas and Chlamydiae in the genital tract of the women presenting in spontaneous early preterm labour. Journal of Medical Microbiology 1987; 24: 253-257.

35. Wahbeh C.J., Hill G.B., Eden R.B., Gall S.A. Intra-amniotic bacterial colonisation in premature labour. American Journal of Obstetrics \& Gynaecology 1984; 148: 739-742.

36. Relier J.P. Perinatal and neonatal infections. Listeriosis. Journal of Antimicrobial Chemotherapy 1979; 5: 51-57.

37. Kass E.H., McCormack W.M., Lin J. Genital Mycoplasms as a cause of excess premature delivery. Transactions of Association of American Physiology 1981; 94: 261-266.

38. King J., Flenady V. Antibiotics for preterm labour with intact membranes (Cochrane Review). In: The Cochrane Library, 2, 1999. Oxford: Update software. 
39. King J., Flenady V. Antibiotics for preterm labour with ruptured membranes (Cochrane Review). In: The Cochrane Library, 2, 1999. Oxford: Update software.

40. S.L. Kenyon, D.J. Taylor, W. Tarnow-Mordi for the ORACLE collaborative group. Broad spectrum antibiotics for spontaneous preterm labour: the ORACLE 11 randomised trial. Lancet 2001; 357: 989-994.

41. Perera J. Microbiological patterns in vaginitis. Ceylon Medical Journal. 1994; 39: 91-94.

42. Eastman N.T. Prematurity from the viewpoint of the obstetrician. American Practitioner 1947; 1: 343-346. 